\title{
Remote operation of the GOLEM tokamak for Fusion Education
}

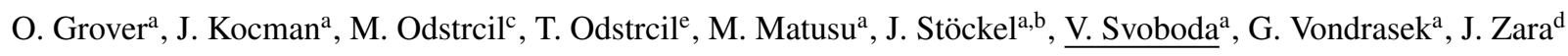 \\ ${ }^{a}$ Faculty of Nuclear Sciences and Physical Engineering CTU Prague, CZ-115 19, Czech Republic \\ ${ }^{b}$ Institute of Plasma Physics AS CR, Prague, CZ-182 21, Czech Republic \\ ${ }^{c}$ University of Southampton, Southampton, SO17 1BJ, UK \\ ${ }^{d}$ Faculty of Electrical Engineering CTU Prague, CZ-166 27, Czech Republic \\ ${ }^{e}$ Max-Planck-Institut für Plasmaphysik, D-85748 Garching, Germany
}

\begin{abstract}
Practically oriented education in the field of thermonuclear fusion is highly requested. However, the high complexity of appropriate experiments makes it difficult to develop and maintain laboratories where students can take part in hands-on experiments in this field of study. One possible solution is to establish centres with specific high temperature plasma experiments where students can visit such a laboratory and perform their experiments in-situ. With the advancements of IT technologies it naturally follows to make a step forward and connect these with necessary plasma physics technologies and thus allow to access even sophisticated experiments remotely. Tokamak GOLEM is a small, modest device with its infrastructure linked to web technologies allowing students to set-up necessary discharge parameters, submit them into a queue and within minutes obtain the results in the form of a discharge homepage.
\end{abstract}

Keywords: Tokamak technology, remote participation, education, nuclear fusion.

\section{Introduction}

Last two decades of advancements in IT technologies offer new possibilities to control experiments remotely. There are two common applications: i) allowing control and data access to complex scientific experiments without the need to be present on the site [1, 2, 3] and ii) providing new educational tools in the form of standalone, relatively simple experiments for teachers and curious students in the field of technical education, opening new strategy in teaching, see e.g. [4], [5]. However, there is still a gap in development of complex, remotely operable experiments for higher education. There are approaches to provide full control of big experiments remotely for researches, like in [6], but education still suffers in this field of study.[a]

The GOLEM tokamak (see e.g. [7]) which has been operated for more than 40 years in the Kurchatov institute near Moscow and in the Institute of Plasma physics in Prague as a scientific facility, recently became an educational device for domestic as well as foreign students, via remote participation/handling. The re-installed tokamak $(R=0.4 \mathrm{~m}, a=0.085 \mathrm{~m})$ operates currently at a modest range of parameters: $B_{t}<0.6 \mathrm{~T}$, I $p<8 \mathrm{kA}$, discharge duration $<20 \mathrm{~ms}$ and with a limited set of diagnostics. A unique feature of this experimental arrangement is the possibility of complete remote handling operation via Internet access. From the client side the tokamak is operated through HTTP or SSH connections, whereby a remote operator can set all the discharge parameters and submit them into a queue and then special software performs the queued discharges according to the submitted requests. Consequently, all data in graphical/raw form are accessible on a special discharge web page. More than 1000 discharges have been performed re-

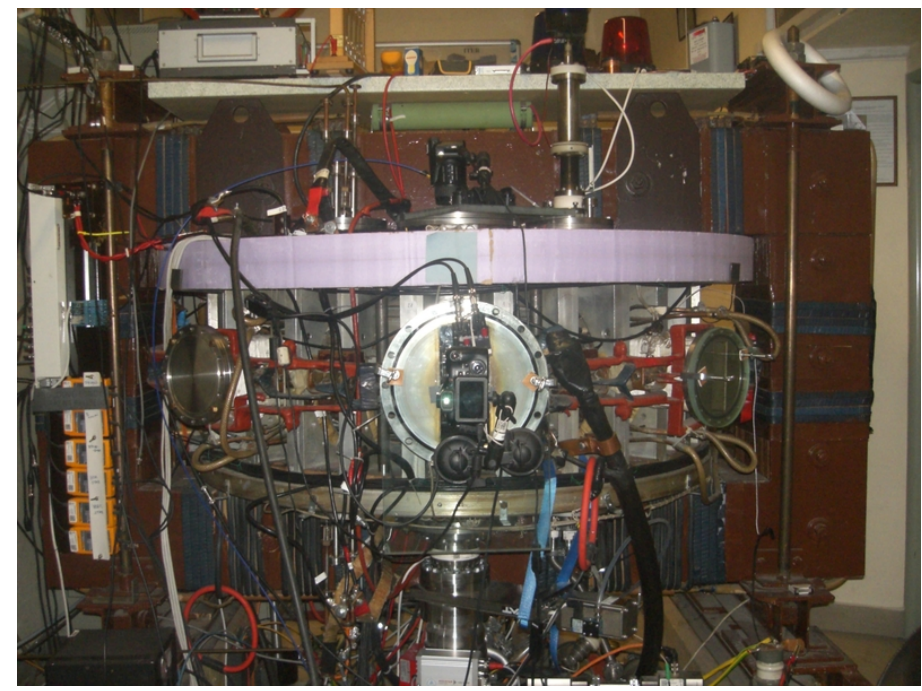

Figure 1: Tokamak GOLEM installed at the Czech Technical University

motely across the borders of the Czech Republic as a part of a remote laboratory practice or a tokamak performance presentation for foreign students in various plasma schools, workshops, lectures and training courses.

The paper organization follows the scenario of the initial student training in the tokamak operation and physics: from initial steps covering acquaintance with the system through the identification of possible education tasks, to the real tokamak control and final data analysis. 


\section{The experimental setup}

The overview of the GOLEM tokamak experiment is presented in Fig. 2 and in a simplified form consists of two parts: i) the tokamak itself with its infrastructure securing vacuum conditions, working gas management, pre-ionization, toroidal magnetic field $B_{t}$ generation for plasma confinement and toroidal electric field $E_{t}$ generation for plasma breakdown and subsequent heating and ii) basic diagnostics consisting of the loop voltage $U_{l}$, toroidal magnetic field $B_{t}$, plasma current $I_{p}$ and visible emission measurements. Both parts are controlled by a computer connected to a web server and thus provides the link between the tokamak and IT technology. On the client side any device capable of reaching the web with a simple web browser is required without need to install any third party software. After necessary authentication and authorization via standard Apache HTTP server configuration [8], the users can choose requested parameters of the plasma discharge in a web-page form, specify a textual comment to the planned discharge and submit their selection into a queue. The parameters available in "menus" of the form cannot harm or damage the facility in any way. The discharge set-ups are later processed and remote participants obtain results in the form of a discharge homepage. For (not only) safety reasons it is not possible to run the tokamak without a local supervisor on site. This supervisor is responsible for initial procedures to start the vacuum pumping of the tokamak vessel and initiate diagnostics and keeping the whole system running during the session. Also special requests (e.g. changing positions of diagnostics, initiating a glow discharge and chamber conditioning) must be requested remotely and finally handled locally. The local supervisor is also responsible for fixing minor technological problems that can appear during the operation.

The educational aspect of the experiment demands that the operation is made as simple as possible and thus the "first level" approach allows to specify required target voltages for charging appropriate capacitor banks and a time delay between their discharging for driving currents in the toroidal magnetic field coils and primary windings of tokamak transformer coil and a time delay between their initiation.

The work-flow of the remote session is depicted in the Fig. 3 having these basic steps (assuming there is an opened session, according to negotiations between the participants and GOLEM tokamak supervision ${ }^{1}$ : i) Session preparation phase, initiating necessary technological processes (evacuating the vessel, starting data diagnostics systems) and eventual vessel conditioning. ii) Web oriented control room, where participants set and submit the parameters simultaneously through the web page form via drop-down menus, see the Technology part menu of the Fig. 2. The control system then stores the request into a SQL database with the flag of waiting request. A virtually unlimited (within the very large capacity of the SQL server) number of requests can be submitted at once and/or during

\footnotetext{
${ }^{1}$ the GOLEM tokamak is not a available 7 day/week and 24 hours/day, the session should be requested
}

the session. iii) Remote operation daemon, process regularly checks the queue of submitted discharge set-ups and executes the requests one by one in a "First In, First Out" manner. iv) Discharge preparation phase, where capacitors are charged, pre-ionization started and working gas introduced into the vessel. v) Discharge, where the thyristors are triggered, delivering current into the appropriate coils and the plasma discharge is performed. During the discharge Data Acquisition Systems (DAS) collect data from various diagnostics. vi) Postdischarge phase, where the acquired data are downloaded to the central server and automated scripts create web pages with reports dedicated to individual DAS systems and all diagnostics.

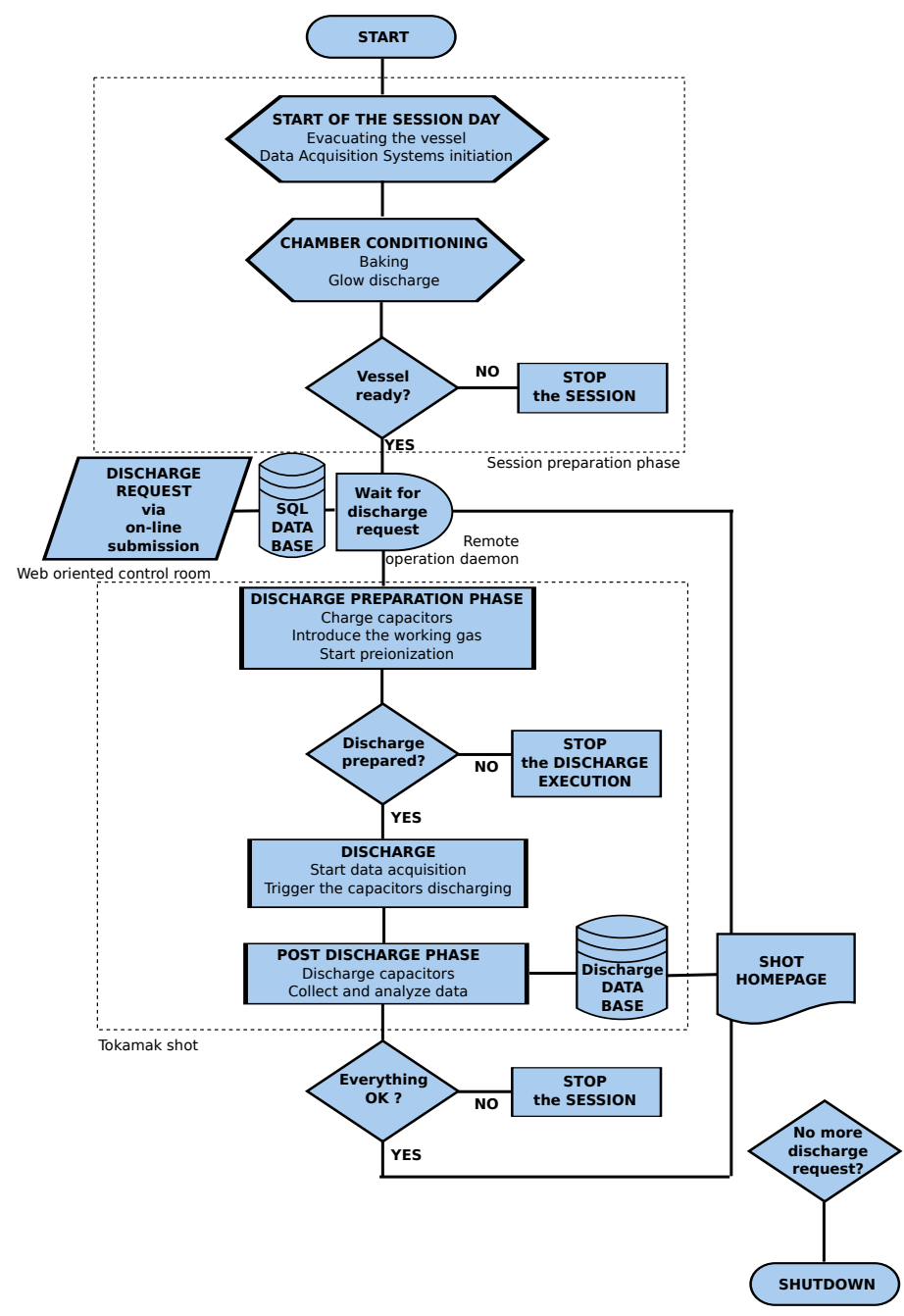

Figure 3: Flowchart diagram of the remote session.

\section{Getting ready for the (remote) tokamak GOLEM control}

Since there is necessity to train inexperienced students in a limited time of the particular training course in tokamak operation, it is desirable to have a set of tools helping students to get familiar with the system in advance. Therefore a variety of methodical materials, a wiki-like system of documentation and 
a tokamak GOLEM virtual simulator have been created for that purpose.

A collection of methodical materials covering various physical and technological aspects of tokamak operation and diagnostics have been prepared: i) Basics of the tokamak operation, optimizing start up phase of the tokamak discharge. ii) Basic tokamak measurements, covering methods of data acquisition and its evaluation. iii) Breakdown studies, determining a critical value of the toroidal electric field needed for mass gas ionization and its consequent breakdown into plasma for given working gas pressure. iv) The Vessel conditioning, to find the best cleaning strategy to minimize the impurity contents of the plasma. v) Isotopic studies, where plasma characteristics are compared in different working gases. vi) Spectroscopy studies, where the intensities of impurity lines are studied. vii) Edge plasma studies, investigating plasma parameters by electric probes different discharges regimes. viii) Besides these, runaway electron, magnetohydrodynamic, plasma flow and other studies are of the next level approach that can be performed on the GOLEM tokamak.

\subsection{The GOLEM web $\mathcal{G}$ GOLEM wiki}

The documentation with wiki-like structure http://golem. fjfi.cvut.cz/wiki/, containing all information about the tokamak GOLEM experiment, including the tokamak set-up and corresponding parameters, the structure of the data acquisition system, the layout of the all diagnostics, reports on all experiments, which have been performed on GOLEM, a library with all relevant publications, etc.

\subsection{The GOLEM control simulator}

Students have the opportunity to learn the basics of GOLEM operation in advance via the virtual interface, which has the same design as the web-based real tokamak control interface (see Fig. 5). The only difference is that tokamak operation is only simulated and the results are generated from the real discharge database of the previous GOLEM tokamak operation (a discharge from the database is selected to have set-up parameters as close as possible to the parameters chosen by the student).

\section{3. $3 D$ virtual model}

In order to improve the visual attractiveness and to provide a catching feedback to remotely connected students, a 3D model of tokamak has been developed and included in respective web pages. While the first version of the $3 \mathrm{D}$ model was created in VRML language (an ISO standard) and required a special browser plug-in to be installed, current version has been built using WebGL language, a cross-platform de facto standard for 3D web applications. This innovation has allowed a direct presentation in majority of common web browsers and much easier integration with further functionalities of simulation parameters and processes. JavaScript interface provides an access to server/database to retrieve real and/or simulated data; it also helps to easily change and enhance a user interface by adding virtual handles, switches and other controls into web page(s).
Without installing any additional software, remote participants can perform various activities with the 3D model: i) Visit all rooms and corridors around the tokamak, see Fig. 4 ii) Study tokamak components by clicking on various virtual parts. iii) Visualize processes inside the tokamak.

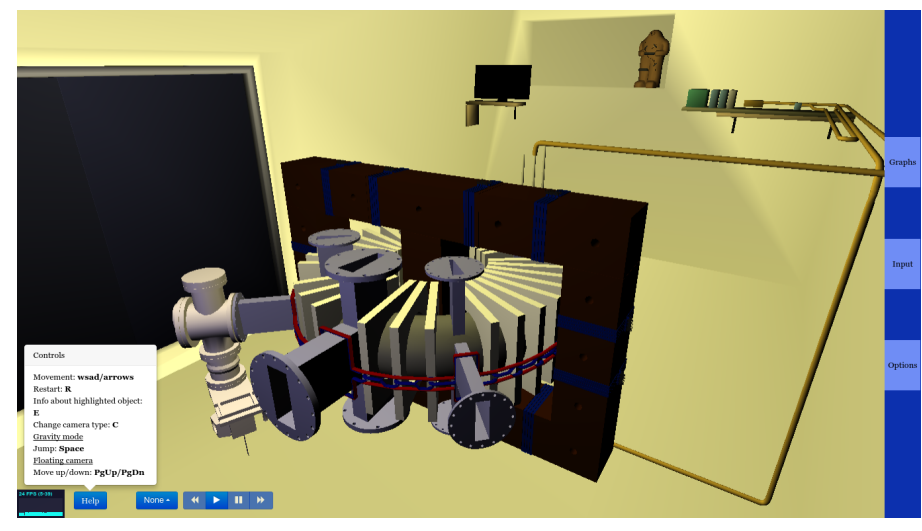

Figure 4: Preview of the virtual 3D model of the tokamak structure

\subsection{Data access}

Data access systems at majority of tokamaks are too complex to be exploited by inexperienced users. Therefore, we have developed a system with the ambition to be intuitive, user-friendly, with appropriate description of the data, so that these can be used by students with only a limited knowledge of data collecting systems. Every predefined variable in the database has a unique web address of the form http://golem.fjfi.cvut.cz/shots/<\#shotno $>/<$ value_name $>$ The signals are converted automatically to the requested format depending on the file-name extension. The raw data format download and their graphical representation as well is a matter of course. It allows easy data access via http protocol through most of the programming languages. Furthermore, scripts and examples for Python, Matlab and IDL are provided that give the options for advanced data processing and data mining. Since the Application Program Interface is based on the html protocol, it is clear, that the human-system interaction is equivalent for remote as well as on-site data manipulation regardless of the user geographical location or the digital system.

\section{The web interface for the remote control of the GOLEM tokamak}

The local as well as the remote control of the GOLEM operation is based on web interface. It is designed to be used by a number of groups and/or users performing their own investigations in the same time. This is possible due to a rather high repetition rate (the discharge every 2 minutes), allowing to perform up to 200 discharges per one day. A control "room" is a set of 8 tabs: i) Home, a welcome address to the users, describing introductory information about the Golem facility. ii) Golem wiki, as described in the part 3.1. iii) Control room, with modular functionality, where users can build up their own layout of 
the experiment or choose from existing .... The web form based system allowing to specify all necessary parameters of the discharge (e.g. $U_{B t}, U_{C D}, T_{C D}$ and $p_{W G}$ ) in the safety operation range and submit into a database oriented queue where it waits for completion. iv) Queue, where users can monitor all submitted discharges with an estimation of the time to completion. v) Live, a tab, visually indicating current status of the tokamak operation, e.g. actual pressure in the vessel, actual status of the device (waiting for the queue call, pre/due/post -discharge phase), charging state of capacitors, status of key relays, etc. vi) Results, a similar database oriented monitor of all submitted set-ups after completion with the link to the corresponding discharge home-pages. vii) IP cameras, a tab with two windows: a) providing a real-time view into the tokamak room b) view of the vessel inside, where users can watch the status of glowing discharge, preionization filament lightening the vessel in the pre-discharge phase and a flash of the plasma during discharge phase. viii) Chat, giving access to the standard preprepared IRC protocol, allowing interactive many-to-many and one-to-one discussions.

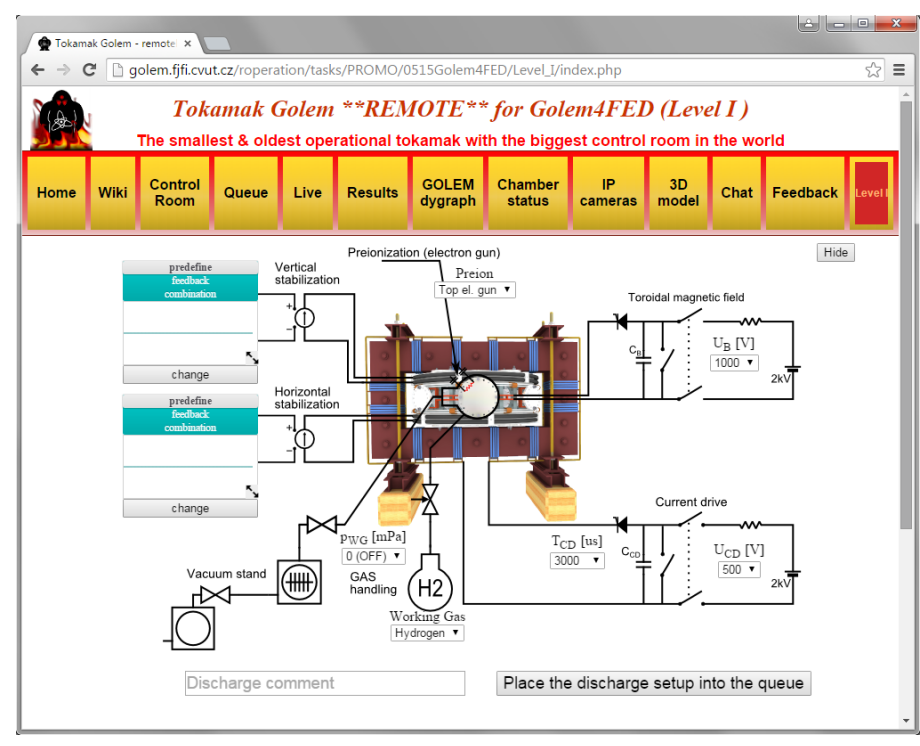

Figure 5: Tokamak Golem web based control room

\subsection{Level organization of the experiment}

Since the tokamak GOLEM is mainly used for educational purposes, it is instructive to allow participants to study the appropriate topics from the basic to the more sophisticated levels. The current organization of the human-machine interface is organized as follows: i) Level "system check", with the purpose to test individual parts, where students can trigger toroidal magnetic field or toroidal electric field separately and observe their particular effect in the diagnostic system. ii) Level "basic tokamak plasma", where the minimum technology required to control the tokamak is used to set-up the tokamak plasma: simultaneous trigger of the toroidal magnetic and electric field, while working gas and pre-ionization tool have been engaged in advance to the trigger. iii) Level "vessel conditioning", where participants can study improved plasma performance regimes after vessel conditioning with the help of baking and the glow discharge cleaning. iv) Level " $E_{t}, B_{t}$ orientation", where the influence of the mutual orientation of the toroidal magnetic as well as electric field on the plasma performance can be examined. v) Level plasma stabilization, where students can set-up via a web interface a pre-defined or feedback plasma position control scenario, see [9].

\subsection{Individual scripts}

Each web page in the discharge database contains a link on the source code used to create this particular page including scripts that were used for data processing, analysis and plotting. Source code of these scripts is entirely open and available to everyone. It makes easy to benchmark or clone these scripts for creation of new user defined diagnostics or analysis that uses any kind of acquired signals or processed data. Moreover, modularity of the system makes it resistant to possible students mistakes and code inconsistencies. The GOLEM database system and supporting libraries (called PyGOLEM) are written in Python, however user-defined diagnostics can be written in any executable script or program. Therefore, the almost unlimited scope of various computer algebraic systems that can be adopted in the system includes Numpy, Matlab, Mathematica, IDL, Octave, Gnuplot, Maple, Science, Perl etc. The user scripts are executed in four subsequent levels and each level executes all its scripts in parallel. The subsequent levels are DAS, Diagnostics, Analysis, Post-analysis. Each script in a given level can depend only on the scripts are values generated in the previous levels. This avoids circular dependencies and allows fast execution.

Available scripts are able to perform a detailed analyses of the all magnetics, spectroscopic and other signals usually in 2 minutes after the discharge. Therefore rather sophisticated analyses are available for users for immediate evaluation and study interesting phenomena.

\section{The web interface for results - discharge homepage}

With respect to the complexity of the tokamak technology, the data access system of the GOLEM tokamak was designed to allow students to get easily oriented within large amount of various data in a limited time. Therefore, most of the variables on the discharge homepage contains links to the wiki system with their detail explanations and correct definitions.

The discharge homepage provides all important information about each discharge and it is divided into specific parts: i) Basic information, listing all the technological parameters of the discharge and consequently acquired physical variables presented in the form of values or time dependent characteristics. An automatic tag system is provided to improve orientation within the large number of discharge in the database and search for discharges with similar setting or plasma behaviour. ii) DAS, presenting all Data acquisition systems presently gathering data in the form of raw voltage signals. iii) Diagnostics, other diagnostic systems, like spectrometer, fast visible light cameras [10] and additional processing of 


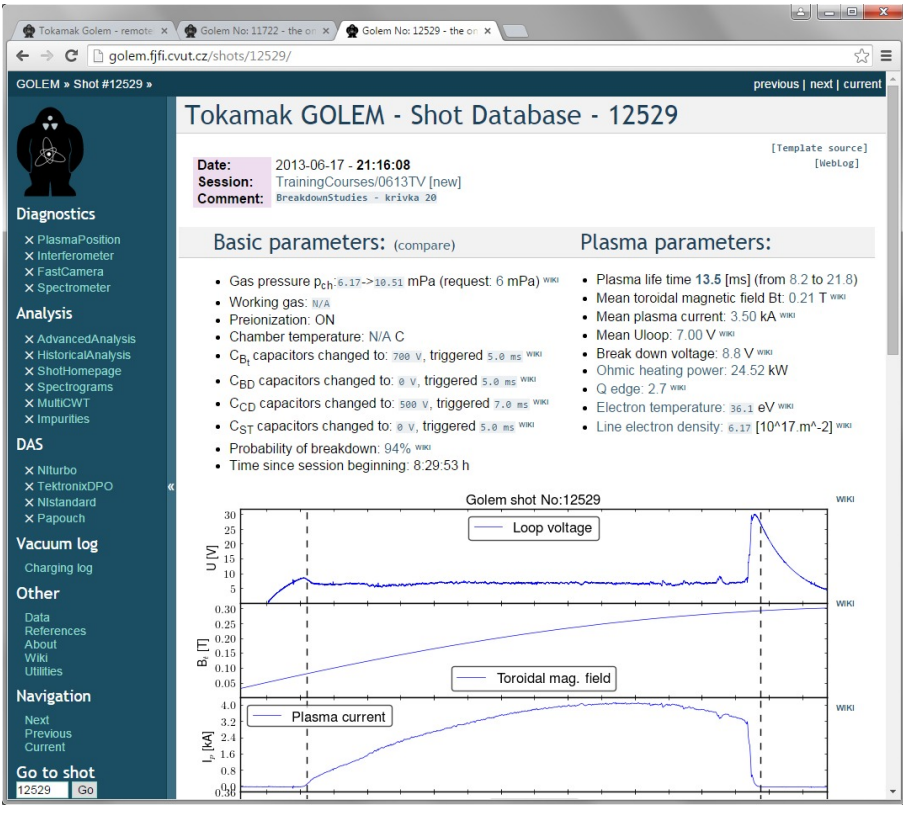

Figure 6: Home page of the GOLEM discharge containing all important informations and references to the diagnostics and and evaluated analysis of these measurements

raw signals from DAS iv) Analysis, recalculated physical values combining multiple diagnostics and DAS with advanced data processing methods and plasma models allow to quantize more complex plasma phenomena such as magnetohydrodynamic modes,reconstruction of the plasma position or time evolution of impurities in plasma discharge. Furthermore, historical analysis show evolution of important plasma parameters over large time periods allowing to evaluate results of different plasma cleaning methods or improved plasma positioning scenarios. v) Analysis tools, PyGolPlot - web based plotting system that allows to quickly compare time evolution of different signals from multiple diagnostics and discharges, GolSQL - a simple web-based data-mining tool designed for fast filtering and comparing scalar variables over a large number of discharges and determination of correlation and dependencies between them, and GolSearch - a web-based tool designed for searching in the discharge database and filter out discharges with given parameters and measured values or close to them.

\section{Conclusions}

Authors present their vision to provide understandable tokamak for education purposes. The education in the field of the basic physics, technology and operation of the tokamaks is now available in-situ as well as remotely with the help of easy configurable html based form and almost instantly obtaining results in the form of discharge homepage. The system is developed with the aim to offer easy and fast insight into the problematic of the control thermonuclear fusion.

Because of limited space, accompanying page [11] have been created with direct link to the all presented components that can be tested - even remote operation of the tokamak. Readers are welcome to contact authors to test the system and colleagues at the universities teaching the physics, technology and operation of tokamaks curricula are encouraged to exploit the GOLEM facility for educational purposes as well.

\section{Acknowledgement}

The project was partially supported by the FUSENET, CTU RVO68407700, IAEA technical contract CRP F1.30.14 on Utilization of the Network of Small Magnetic Confinement Fusion Devices for Mainstream Fusion Research. The opinions expressed by authors do not necessarily represent the positions of the European Commission neither IAEA. This work was partially supported by the Grant Agency of the Czech Technical University in Prague, grant SGS15/164/OHK4/2T/14.

Authors express their gratitude to dr. Gergo Pokol from Budapest University of Technology (BUTE) for his valuable contribution to the project developing first methodical materials and testing it with BUTE students

\section{References}

[1] Steve F Horne, Martin Greenwald, Tom W Fredian, Ian H Hutchinson, Brian Louis Labombard, Josh Stillerman, Yuichi Takase, Stephen M Wolfe, Thomas A Casper, David N Butner, et al. Remote control of alcator c-mod from lawrence livermore national laboratory. Fusion Science and Technology, 32(1):152-160, 1997.

[2] Bill B McHarg Jr, TA Casper, S Davis, and D Greenwood. Tools for remote collaboration on the DIII-D national fusion facility. Fusion Engineering and Design, 43(3):343-355, 1999.

[3] DP Schissel. A vision for a collaborative control room for ITER. Fusion Engineering and Design, 83(2):539-544, 2008.

[4] Frantisek Schauer, Frantisek Lustig, Jiri Dvorak, and Miroslava Ozvoldova. An easy-to-build remote laboratory with data transfer using the Internet School Experimental System. European Journal Of Physics, 29(4):753-765, JUL 2008.

[5] Ananda Maiti and Balakrushna Tripathy. Remote Laboratories: Design of Experiments and Their Web Implementation. Educational Technology $\mathcal{E}$ Society, 16(3):220-233, JUL 2013.

[6] Wei Zheng, Ming Zhang, Guang Li, Jing Zhang, and Ge Zhuang. ServiceOriented Remote Operation System for J-TEXT Tokamak. Ieee Transactions On Plasma Science, 42(3, 1, SI):477-481, MAR 2014.

[7] V. Svoboda, B. Huang, J. Mlynar, G.I. Pokol, J. Stockel, and G Vondrasek. Multi-mode Remote Participation on the GOLEM Tokamak. Fusion Engineering and Design, 86(6-8):1310-1314, 2011.

[8] Apache Software Foundation, 2016.

[9] V. Svoboda, J. Kocman, O. Grover, J. Krbec, and J. Stockel. Remote operation of the vertical plasma stabilization @ the golem tokamak for the plasma physics education. Fusion Engineering and Design, accepted for publication, 2015. Proceedings of the 28th Symposium On Fusion Technology (SOFT-28); San Sebastian, 2014.

[10] T. Odstrcil, M. Odstrcil, O. Grover, V. Svoboda, I. Duran, and J. Mlynar. Low cost alternative of high speed visible light camera for tokamak experimentsa). Review of Scientific Instruments, 83(10):-, 2012.

[11] Tokamak GOLEM. Tokamak GOLEM for FED 2015, accompanying page. http://golem.fjfi.cvut.cz/15FED, 2015. 
Tokamak technology setup

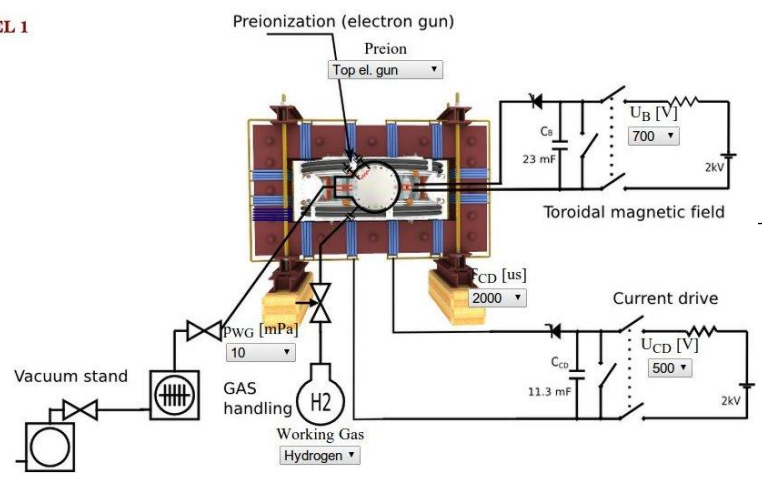

Basic plasma diagnostics

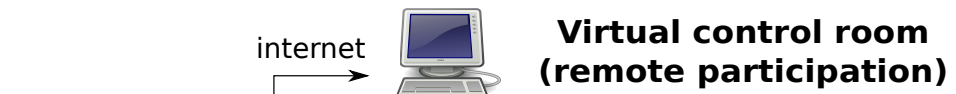

WWW control interface

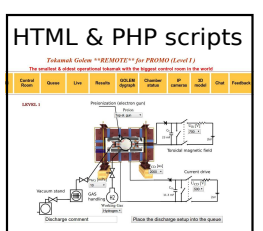

SSH control interface

WINDOWS via putty

\begin{tabular}{|c|c|}
\hline VINDOWS via putty & Data handling \\
\hline 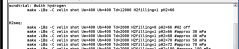 & *wget \\
\hline$z=$ & *gnuplot \\
\hline 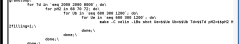 & *idl \\
\hline$=-\ldots$ & *mathematica \\
\hline $\begin{array}{l}\text { LINUX via ssh } \\
\text { or ssh }+X \text { tunnel } \\
\text { (advanced mode) }\end{array}$ & $\begin{array}{l}* \text { matlab } \\
* \text { etc... }\end{array}$ \\
\hline
\end{tabular}

Data presentation

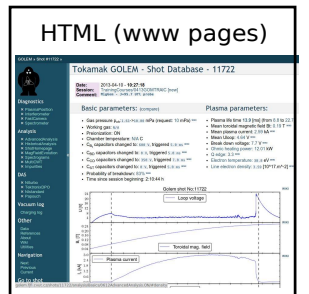

OpenSSH server

(advanced mode)
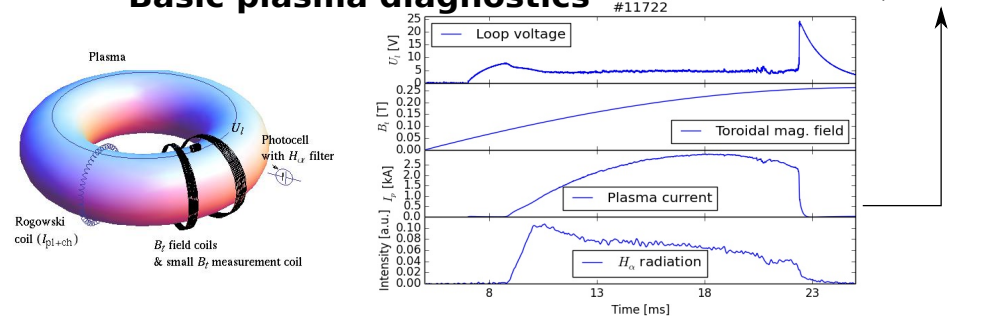

Figure 2: The global schematic overview of the tokamak GOLEM experiment 